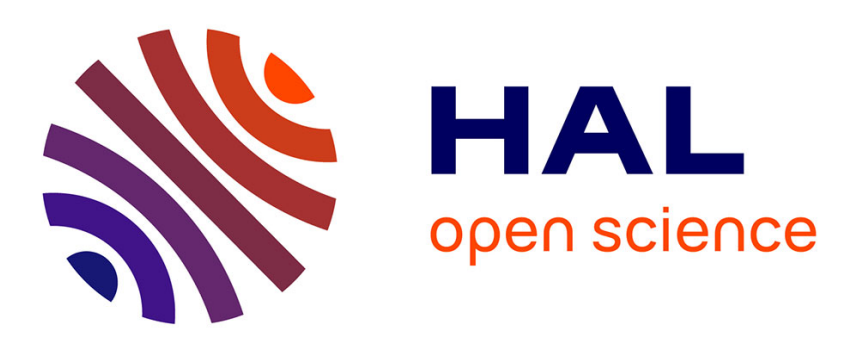

\title{
Online Motion Detection in High Angular Resolution Diffusion Imaging
}

\author{
Emmanuel Caruyer, Iman Aganj, Christophe Lenglet, Guillermo Sapiro, \\ Rachid Deriche
}

\section{- To cite this version:}

Emmanuel Caruyer, Iman Aganj, Christophe Lenglet, Guillermo Sapiro, Rachid Deriche. Online Motion Detection in High Angular Resolution Diffusion Imaging. International Symposium on Biomedical Imaging: From Nano to Macro, Mar 2011, Chicago, United States. inria-00559904v2

\section{HAL Id: inria-00559904 https://inria.hal.science/inria-00559904v2}

Submitted on 22 Feb 2011

HAL is a multi-disciplinary open access archive for the deposit and dissemination of scientific research documents, whether they are published or not. The documents may come from teaching and research institutions in France or abroad, or from public or private research centers.
L'archive ouverte pluridisciplinaire HAL, est destinée au dépôt et à la diffusion de documents scientifiques de niveau recherche, publiés ou non, émanant des établissements d'enseignement et de recherche français ou étrangers, des laboratoires publics ou privés. 


\title{
ONLINE MOTION DETECTION IN HIGH ANGULAR RESOLUTION DIFFUSION IMAGING
}

\author{
Emmanuel Caruyer ${ }^{1}$, Iman Aganj ${ }^{2}$, Christophe Lenglet ${ }^{3,2}$, Guillermo Sapiro ${ }^{2}$, Rachid Deriche ${ }^{1}$ \\ ${ }^{1}$ Athena Project-Team, INRIA Sophia Antipolis - Méditerranée, France \\ ${ }^{2}$ Department of Electrical and Computer Engineering, University of Minnesota \\ ${ }^{3}$ Center for Magnetic Resonance Research, Department of Radiology, U of Minnesota Medical School
}

\begin{abstract}
The orientation distribution function (ODF) can be reconstructed online incrementally from diffusion-weighted MRI with a Kalman filtering framework. This online reconstruction can provide real-time feedback to the practitioner, especially appreciated for long acquisition protocols typical in Q-ball imaging. On top of the Kalman filter, we propose a method to evaluate online the reconstruction accuracy of the estimated ODF in constant solid angle. In addition, monitoring the residuals of the Kalman filter, we design, based on statistical tests, two algorithms for online detection of subject motion. The proposed techniques, tested on real and synthetic data under various experimental conditions, can detect rotation by angle less than $3^{\circ}$.
\end{abstract}

Index Terms - motion estimation, error propagation, online model estimation, Q-ball imaging (qbi), orientation distribution function (odf).

\section{INTRODUCTION}

In spite of its great potential for clinical applications, the widespread use of high angular resolution diffusion imaging is still hindered by the severe acquisition time limitations in clinical set-up. Among challenges, patients are likely to move their head during long acquisitions. Although motion can be corrected through coregistration in a post-processing step $[1,2]$, this might increase partial voluming effects, due to the coarse spatial resolution of diffusion weighted (DW) images and the interpolation in the registration procedure. Being able to detect motion online, the scanner operator could be issued a warning, and take a decision accordingly.

Recently, the group of Poupon and colleagues proposed a solution based on Kalman filtering for an incremental reconstruction of the diffusion tensor and the regularized ODF [3]. The latter was further corrected by Deriche and colleagues [4], so that the incrementally reconstructed ODF equals the solution computed off-line. Meanwhile, the ODF definition in use within the Q-ball imaging community has been updated lately to match the marginal probability of diffusion, namely the ODF in constant solid angle [5, 6], and we have presented an online Kalman filtering solution to reconstruct this mathematically correct ODF, with the suggestion that motion could also be detected online based on the residuals of this Kalman filter [7].

In this article, we present three contributions related to the problem of online motion detection in HARDI. First, we develop a proper error propagation accounting for the non-linear transform on the diffusion signal. Then, on the top of the Kalman filter, we develop two motion detection algorithms, based on the monitoring of residuals, and compare them in the experimental part, on synthetic and real data.

\section{METHODS}

\subsection{Incremental reconstruction of the solid angle ODF}

The ODF, $\psi$, is the probability for a water molecule to diffuse along a given direction $\mathbf{u}$, in a constant solid angle. It is defined from the diffusion ensemble average propagator $P$ as

$$
\psi(\mathbf{u})=\int_{0}^{\infty} P(r \mathbf{u}) r^{2} \mathrm{~d} r .
$$

Under the assumption of a mono-exponential decay of the diffusion signal $S$, the ODF $\psi$ can be obtained from $S$ as

$$
\psi(\mathbf{u})=\frac{1}{4 \pi}+\frac{1}{16 \pi^{2}} \mathrm{FRT}\left\{\nabla_{b}^{2} \ln \left(-\ln \frac{S(q \mathbf{u})}{S(\mathbf{q}=\mathbf{0})}\right)\right\},
$$

where FRT denotes the Funk-Radon transform, and $\nabla_{b}^{2}$ the Laplace-Beltrami operator [5].

The ODF reconstruction can be implemented using the symmetric, real and orthonormal spherical harmonic basis as in [8] to describe the transformed signal $y=\ln (-\ln S / S(\mathbf{0}))$ [5], since both the Funk-Radon transform and the LaplaceBeltrami operations in Eq. 2 have close-form matrix expressions, $\mathbf{F}$ and $\mathbf{L}$ respectively, in the spherical harmonic basis. In this basis, Eq. 2 is expressed as

$$
\hat{\mathbf{c}}=\frac{1}{2 \sqrt{\pi}} \mathbf{e}_{\mathbf{1}}+\frac{1}{16 \pi^{2}} \mathbf{F L} \mathbf{c},
$$

where $\hat{\mathbf{c}}$ and $\mathbf{c}$ are the vector of spherical harmonic coefficients that describe $\psi$ and $y$, respectively, and $\mathbf{e}_{\mathbf{1}}=$ $\left[\begin{array}{lllll}1 & 0 & 0 & \cdots & 0\end{array}\right]^{\mathrm{T}}$. 
The computation of the spherical harmonic coefficients $\mathbf{c}$ describing $y$ from a series of measurements

$$
y[k]=\ln \left(-\ln \frac{S[k]}{S(\mathbf{0})}\right), k=1 \ldots N,
$$

where $S[k]=S(q \mathbf{u}[k])$ is the signal sampled at discrete positions $\mathbf{u}[k]$ on the unit sphere, is implemented minimizing

$$
M(\mathbf{c})=(\mathbf{y}-\mathbf{B} \mathbf{c})^{\mathrm{T}} \boldsymbol{\Sigma}^{-1}(\mathbf{y}-\mathbf{B} \mathbf{c})+\lambda \mathbf{c}^{\mathrm{T}} \mathbf{L} \mathbf{c},
$$

where $\boldsymbol{\Sigma}$ is the covariance matrix of $\mathbf{y}$, and $\mathbf{B}$ is the spherical harmonic design matrix [8]. The second term is a LaplaceBeltrami regularization constraint on the fitted signal. We presented an incremental solution for the minimization of Eq. 5 in [7], using the Kalman filter:

$$
\left\{\begin{aligned}
\mathbf{c}[0] & =\mathbb{E}[\mathbf{c}[0]] \\
\tilde{\mathbf{P}}[0] & =\mathbb{E}\left[(\mathbf{c}-\mathbf{c}[0])(\mathbf{c}-\mathbf{c}[0])^{\mathrm{T}}\right] \\
\mathbf{P}[0] & =\left(\tilde{\mathbf{P}}_{0}^{-1}+\lambda \mathbf{L}\right)^{-1} \\
\mathbf{P}[k] & =(\mathbf{I}-\mathbf{g}[k] \mathbf{B}[k]) \mathbf{P}[k-1] \\
\mathbf{V}[k] & =\mathbf{B}[k] \mathbf{P}[k-1] \mathbf{B}[k]^{\mathrm{T}}+\sigma^{2}[k] \\
\mathbf{g}[k] & =\mathbf{P}[k-1] \mathbf{B}[k]^{\mathrm{T}} \mathbf{V}[k]^{-1} \\
\gamma[k] & =y[k]-\mathbf{B}[k] \mathbf{c}[k-1] \\
\mathbf{c}[k] & =\mathbf{c}[k-1]+\mathbf{g}[k] \gamma[k]
\end{aligned}\right.
$$

In this article, we emphasize that the $\sigma^{2}[k]$ account for the uncertainty in the DW measurements $S[k]$, as well as for the distortion introduced by the non-linear transform. They can be calculated through error propagation,

$$
\delta y=\frac{\partial y}{\partial S} \delta S=\frac{1}{S \ln (S / S(\mathbf{0}))} \delta S .
$$

Provided that separate measurements are uncorrelated, the diagonal elements $\sigma^{2}[k]$ of $\boldsymbol{\Sigma}$, the covariance matrix of $\mathbf{y}$, are

$$
\sigma^{2}[k]=\frac{\operatorname{Var}(S[k])}{S[k]^{2} \ln ^{2}(S[k] / S(\mathbf{0}))} .
$$

The expected covariance of the estimated spherical harmonic coefficients $\mathbf{c}[k]$ is the matrix $\mathbf{P}[k]$ computed by the Kalman filter. Then the covariance associated to the estimate of the spherical harmonic coefficients describing the ODF is given by $\operatorname{Cov}(\hat{\mathbf{c}}[k])=\mathbf{F}^{\mathrm{T}} \mathbf{L}^{\mathrm{T}} \mathbf{P}[k] \mathbf{L F}$. This result generalizes the case where $\sigma$ was considered as constant [7], and is validated in the experimental section 3.2.1.

\subsection{Motion detection}

Diffusion imaging is a modality particularly prone to motion artifacts, because of the large gradient pulses needed to encode diffusion, and the long acquisition time to sample the Qspace. The use of echo-planar imaging combined with echo navigators, now the gold-standard for clinical diffusion measurements, has considerably reduced such artifacts [9]. However, although each DW image looks unaltered, motion occurring at an instant $\theta$ may introduce misregistration of subsequent images, and then change the frame of reference with respect to which the $b$-matrices are defined.
Since we are here interested in detecting motion, we assume that these effects induced by motion can be summarized as a change in the vector $\mathbf{c}_{\mathbf{x}}$ of spherical harmonic coefficients describing $\ln \left(-\ln S_{\mathbf{x}}(\mathbf{q}) / S_{\mathbf{x}}(\mathbf{0})\right)$ at voxel position $\mathbf{x}$ :

$$
\mathbf{c}_{\mathbf{x}}[k \geq \theta]=\mathbf{c}_{\mathbf{x}}[k<\theta]+\mathbf{p}_{\mathbf{x}} .
$$

Detecting this jump in the state vector $\mathbf{c}[k]$ of the Kalman filter consists in discriminating between two hypotheses:

- $\mathcal{H}_{0}$ : no change occurred in the state vector,

- $\mathcal{H}_{1}$ : a jump $\mathbf{p}$ in the state vector occured at instant $\theta$.

As a consequence of a change in the state vector at time $k=$ $\theta$, the prediction error for subsequent iterations of the Kalman filter will no longer be zero-mean. As it has been shown in [10], this can be decomposed as

$$
\gamma[k]=\mathbf{G}(k, \theta) \mathbf{p}+\gamma_{1}[k],
$$

where $\gamma_{1}$ is zero-mean Gaussian distributed with covariance $\mathbf{V}[k] . \mathbf{G}(k, \theta)$ represents the propagation of a jump at time $\theta$, to the prediction error at time $k$, and can be precomputed [10] from the observation matrix $\mathbf{B}[k]$ and the Kalman gain $\mathbf{g}[k]$ :

$$
\left\{\begin{array}{l}
\mathbf{G}(k, \theta)=\mathbf{B}[k]\left(\mathbf{I}-\sum_{j=\theta}^{k-1} \mathbf{g}[j] \mathbf{G}(j, \theta)\right), \\
\mathbf{G}(k, k)=\mathbf{B}[k] .
\end{array}\right.
$$

In the sequel we present two methods for the detection based on the monitoring of residuals.

\subsubsection{A direct approach}

The expected value of $\gamma[k]^{2}-V[k]$ over the whole volume may increase significantly at time $k=\theta$, as at each voxel position $\mathbf{x}$, a jump of magnitude $\mathbf{p}_{\mathbf{x}}$ is observed in the state vector. We can estimate the mean squared value of $\mathbf{p}_{\mathbf{x}}$ over the volume of interest by averaging $\gamma_{\mathbf{x}}[k]^{2}$. A direct approach for the detection of motion is to apply a simple thresholding on this statistic. Because of its low delay, this test might be a good solution in a clinical context. We propose in the next section a more robust approach, since there are several situations for which this solution is likely to fail, as it will be shown in the experimental part.

\subsubsection{Generalized likelihood ratio test}

Beyond the simple test of the direct approach, we propose a detection based on a general likelihood ratio test (GLRT) [10]. The propagation of a jump $\mathbf{p}$ on the residuals at time $\theta, \theta+1, \ldots, k$ in Eq. 10 is a linear system. Provided that we have sufficient observations since instant $\theta$, and we have an estimate $\tilde{\theta}$ of the instant of motion, we can estimate $\hat{\mathbf{p}}(k ; \tilde{\theta})$ through weighted least squares. This corresponds to the maximum likelihood estimate of $\mathbf{p}$, provided that all densities are 


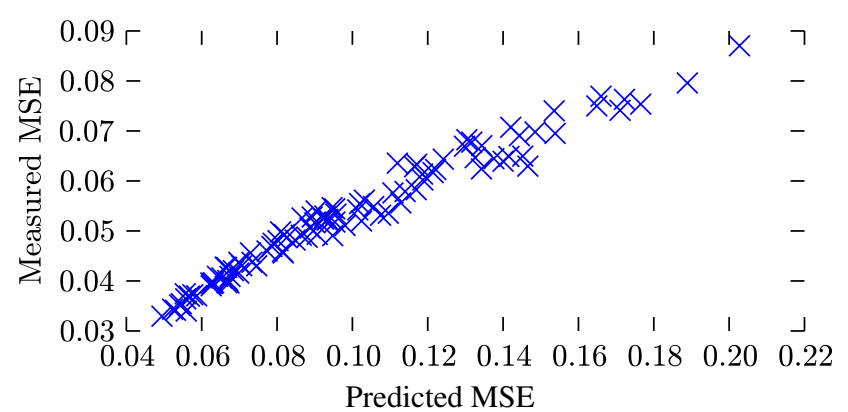

Fig. 1. Predicted reconstruction accuracy versus observed MSE.

Gaussian. Precisely, the value of the likelihood at $\hat{\mathbf{p}}(k ; \tilde{\theta})$ is useful to decide whether a motion occurred or not. The main drawback of this algorithm is its intrinsic delay. But this memory effect could be a desirable property for the detection of very small motion, or the detection of motion occurring at the first few iterations of the Kalman filter.

\section{EXPERIMENTS AND RESULTS}

We implemented the incremental reconstruction using Kalman filtering, together with reconstruction accuracy estimation and motion detection. Quantitative analysis of both algorithms (direct and GLRT) was performed on real and simulated data.

\subsection{On demand reconstruction accuracy}

To check whether the covariance matrix of the Kalman filter is a good indication for the reconstruction accuracy, we tested the Kalman filter on a set of 100 synthetic diffusion propagators, generated using the multi-tensor model, for 1-, 2- and 3-tensors, with trace and FA typical of white matter diffusivity. We estimated the MSE of $\hat{\mathbf{c}}$, and compared it to the trace of the covariance matrix $\mathbf{F}^{\mathrm{T}} \mathbf{L}^{\mathrm{T}} \mathbf{P} \mathbf{L F}$ given by the Kalman filter.

The results of Monte-Carlo simulations are presented on Fig. 1. Each point on this figure represents the result for a given diffusion propagator, estimated from 1000 repetitions. It first appears that the reconstruction accuracy is highly variable from one diffusion propagator to another. This is an evidence that the reconstruction accuracy depends on the diffusion properties, and consequently on the measured intensities.

In addition, the predicted mean squared error is strongly correlated to the empirical mean squared error. This index given by the Kalman filter, with a proper propagation of noise, is a powerful indicator for the quality of the reconstructed ODF, which can be computed online.

\subsection{Motion detection: sensitivity and specificity}

\subsubsection{Monte-Carlo simulations}

To have full control on the motion amplitude and time, we have simulated the formation of DW images, with a motion during acquisition. We first fit a HARDI profile from a series of $200 \mathrm{DW}$ images of a still subject, acquired on 3T Siemens scanner at the Center for Magnetic Resonance Research, University of Minnesota, with 200 encoding directions, $b=1000 \mathrm{~s} / \mathrm{mm}^{2}, 64$ slices with $2 \mathrm{~mm}$ thickness, 25 $b=0 \mathrm{~s} / \mathrm{mm}^{2}$ images, $128 \times 128$ image matrix, TE $=90 \mathrm{~ms}$ and $\mathrm{TR}=8500 \mathrm{~ms}$. For the rigid transform of interest, we extract the rotation component and apply it locally to the diffusion signals. From the baseline image, and the rotated diffusion signals, we synthesise DW images on which we apply the rigid transform. Finally, we corrupt them by Rician noise.

For repeated simulations of scan acquisitions with and without motion, we evaluate the true positive rate $(\mathrm{TPR}=$ \#positives / \#positives detected), also known as sensitivity, as well as the false positive rate (FPR $=\#$ mislabeled negatives/\#negatives). While allowing FPR $\leq 1 \%$, we computed the TPR achieved with both algorithms, while changing various parameters, such as: delay for the detection, instant of motion, SNR, and motion magnitude. Unless explicitly modified, the experimental conditions for all experiments were a rotation around the left-right axis by an angle of 3 degrees, SNR $=20$, motion instant $\theta=20$ and a delay $k-\theta=10$. Result on the detection performance are plotted on Fig. 2, for 400 negatives (simulations without motion) and 400 positives (simulations with motion). The monitoring of residuals is limited to 200 voxels randomly selected within the brain to meet the real-time constraint.

Both algorithms (direct approach and GLRT) were tested in severe experimental conditions: noisy data, small and early motion, and small delay. Both techniques show good results (Fig. 2) in detecting motion. As expected, GLRT is more robust to noise, and performs better in detecting small motion, since it is calculated from a series of residuals. We can also detect motion occurring at the very beginning of a diffusion MRI acquisition: this is a consequence of the memory implemented in the calculation of the likelihood ratio. The more direct approach is slightly less sensitive in noisy conditions, but still can detect motion. Because of its low delay, we suggest to use it in parallel with the GLRT.

\subsubsection{A real data acquisition}

We also validate our methods on a real dataset, with the same imaging parameters as above. During the acquisition, the subject was asked to slightly tilt his head after 80 images were acquired. The motion was a posteriori identified as a rotation of $20^{\circ}$ about the $z$-axis (see Fig. 3). The detection algorithms could detect this motion: with a delay of 2 acquisitions for the GLRT, and with no delay for the direct approach. 

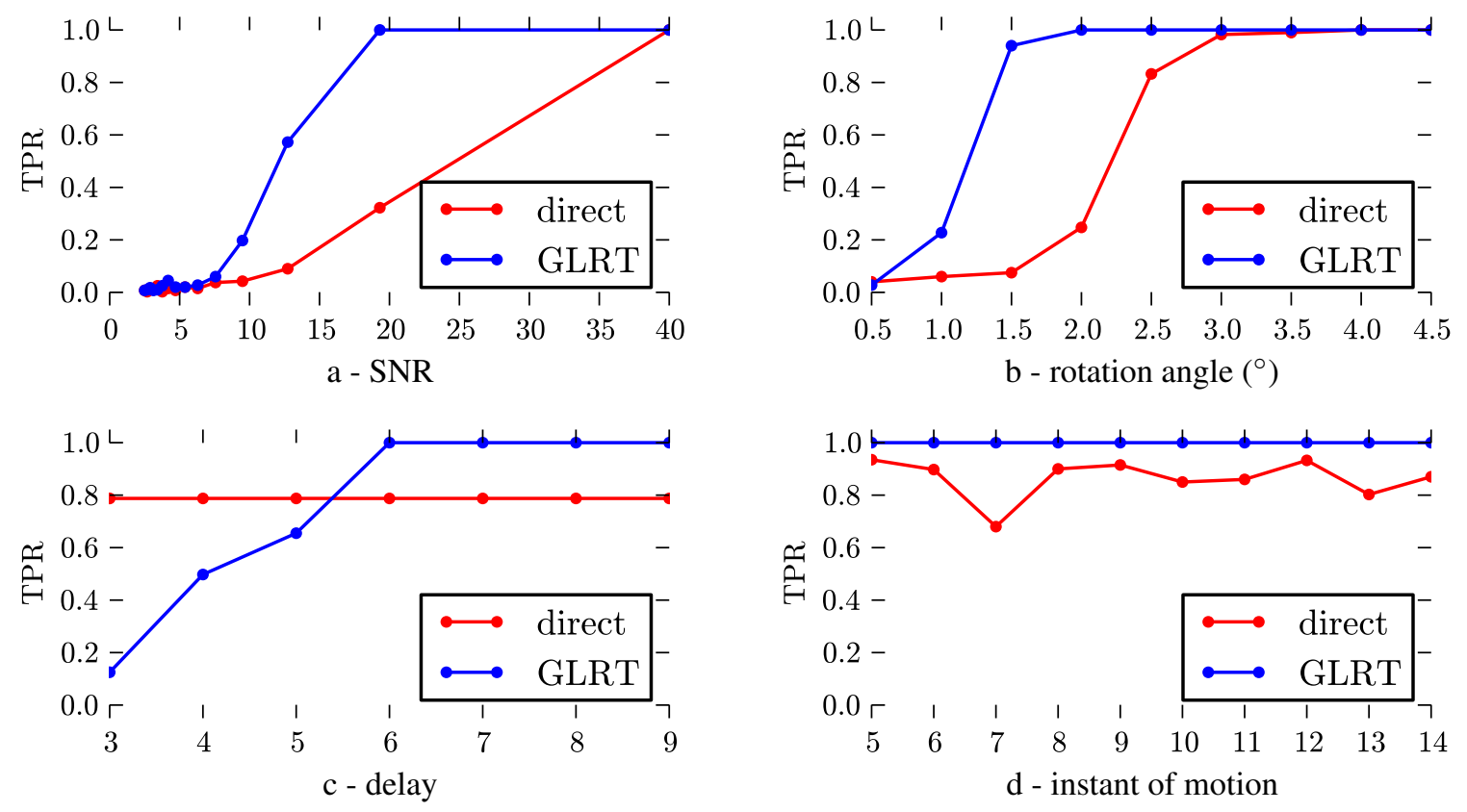

Fig. 2. TPR achieved for FPR $=1 \%$. Each subplot represents the results when changing one experimental parameter.
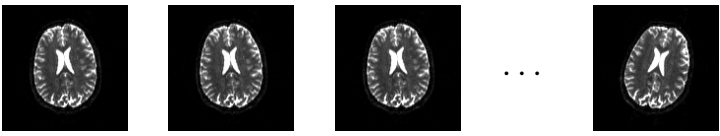

Fig. 3. A real acquisition: the subject was asked to move his head.

\section{CONCLUSION}

We have developed an error propagation scheme, as well as two motion detection algorithms, on a framework for the online processing of high angular resolution diffusion images. We are able to detect small motions (rotation under $3^{\circ}$ ). This contribution is unique at aiming at assisting the scanner operator in real-time, providing clinical information to address reconstruction accuracy and the detection of motion.

Acknowledgements: Work was partly supported by the CD-MRI INRIA Associate Team program, NIH (grants R01 EB008432, P41 RR008079, P30 NS057091), and the University of Minnesota Institute for Translational Neuroscience.

\section{REFERENCES}

[1] G. K. Rohde, A. S. Barnett, P. J. Basser, S. Marenco, and C. Pierpaoli, "Comprehensive approach for correction of motion and distortion in diffusion-weighted MRI," Magn. Reson. Med., vol. 51, no. 1, pp. 103-114, January 2004.

[2] A. Barmpoutis, B. C. Vemuri, and J. R. Forder, "Registration of high angular resolution diffusion MRI images using 4th order tensors," in MICCAI, Berlin, Heidelberg, 2007, pp. 908-915.
[3] C. Poupon, A. Roche, J. Dubois, J.-F. Mangin, and F. Poupon, "Real-time MR diffusion tensor and q-ball imaging using Kalman filtering," Med. Image Anal., vol. 12, no. 5, pp. 527534, 2008, Special issue on MICCAI 2007.

[4] R. Deriche, J. Calder, and M. Descoteaux, "Optimal real-time q-ball imaging using regularized Kalman filtering with incremental orientation sets," Med. Image Anal., vol. 13, no. 4, pp. 564-579, August 2009.

[5] I. Aganj, C. Lenglet, G. Sapiro, E. Yacoub, K. Ugurbil, and N. Harel, "Reconstruction of the ODF in single and multiple shell q-ball imaging within constant solid angle," Magn. Reson. Med., vol. 64, no. 2, pp. 554-566, 2010.

[6] A. Tristán-Vega, C.-F. Westin, and S. Aja-Fernández, "Estimation of fiber orientation PDF in HARDI," NeuroImage, vol. 47, no. 2, pp. 638-650, 2009.

[7] E. Caruyer, I. Aganj, R. L. Muetzel, C. Lenglet, G. Sapiro, and R. Deriche, "Online ODF reconstruction in constant solid angle and its application to motion detection in HARDI," in ISBI, apr 2010.

[8] M. Descoteaux, E. Angelino, S. Fitzgibbons, and R. Deriche, "Regularized, fast, and robust analytical q-ball imaging," Magn. Reson. Med., vol. 58, no. 3, pp. 497-510, 2007.

[9] D. Le Bihan, C. Poupon, A. Amadon, and F. Lethimonnier, "Artifacts and pitfalls in diffusion MRI," J Magn Reson Imaging, vol. 24, no. 3, pp. 478-88, 2006.

[10] A. Willsky and H. Jones, "A generalized likelihood ratio approach to the detection and estimation of jumps in linear systems," Automatic Control, IEEE Transactions on, vol. 21, no. 1, pp. 108 - 112, feb 1976. 